\title{
PICTORIAL INTERLUDES
}

\section{Perinatal lethal osteogenesis imperfecta}

\section{S Moosa}

Division of Human Genetics, National Health Laboratory Service and University of the Witwatersrand, Johannesburg S Moosa, MB BCh, DCH (SA)

Corresponding author: S Moosa (shahidamoosa@gmail.com)

Osteogenesis imperfecta (OI) is a heterogeneous group of genetic bone disorders that are characterised by decreased bone mass, increased bone fragility and susceptibility to fractures. The severe, perinatal lethal form (Type II) (OMIM 166210) is characterised by bone fragility, with perinatal fractures, severe bowing of long bones, undermineralisation, and death in the perinatal period owing to respiratory insufficiency. The overall prevalence of OI Type II is unknown. There are three subtypes of OI Type II (A, B and C) that are characterised by different radiological features, and may be caused by different genetic faults. Two fetuses with OI Type IIA are presented.

S Afr J Rad 2012;16(4):141-142. DOI:10.7196/SAJR.710

Osteogenesis imperfecta (OI) is a heterogeneous group of genetic bone disorders that are characterised by decreased bone mass, increased bone fragility and susceptibility to fractures. The severe, perinatal lethal form (Type II) (OMIM 166210) is characterised by bone fragility, with perinatal fractures, severe bowing of long bones, undermineralisation, and death in the perinatal period owing to respiratory insufficiency. The overall prevalence of OI Type II is unknown. There are three subtypes of OI type II (A, B and $C$ ) that are characterised by different radiological features, and may be caused by different genetic faults. ${ }^{1}$ Two fetuses with OI Type IIA are presented. As there is no molecular genetic testing currently available

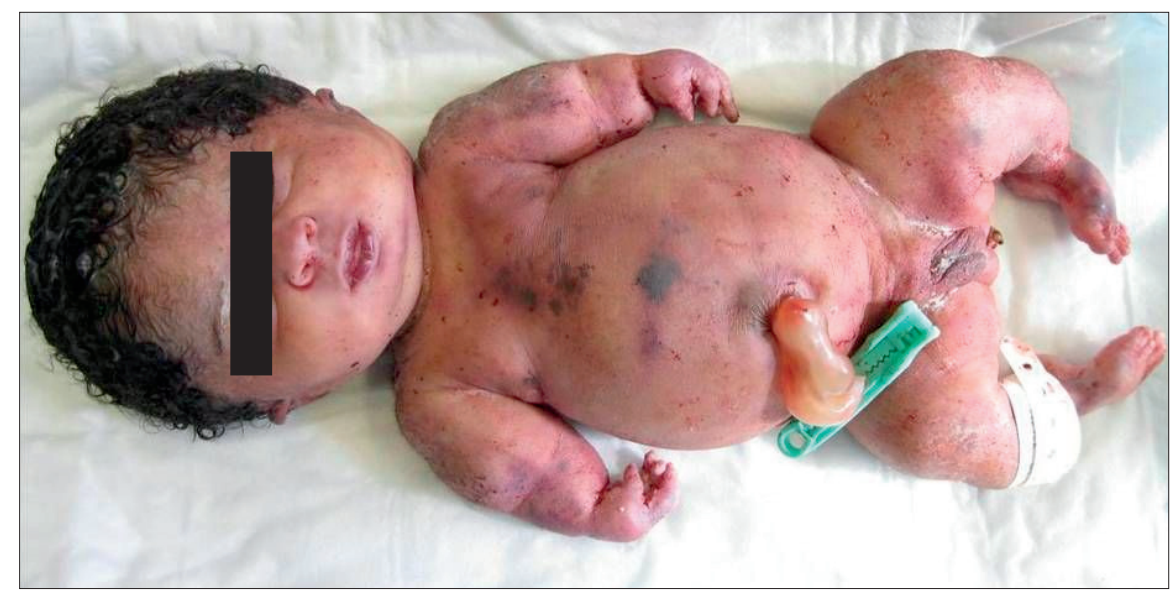

Fig. 1. A female fetus was stillborn at 34 weeks' gestation, with a birth length less than the 3 rd centile and an upper:lower segment ratio of 2.25 (increased). The following dysmorphic features were noted: soft skull, blue sclera, prominent beaked nose, small mouth, short upper limbs (both rhizomelic and mesomelic segments) and bowed lower limbs. 


\section{PICTORIAL INTERLUDES}

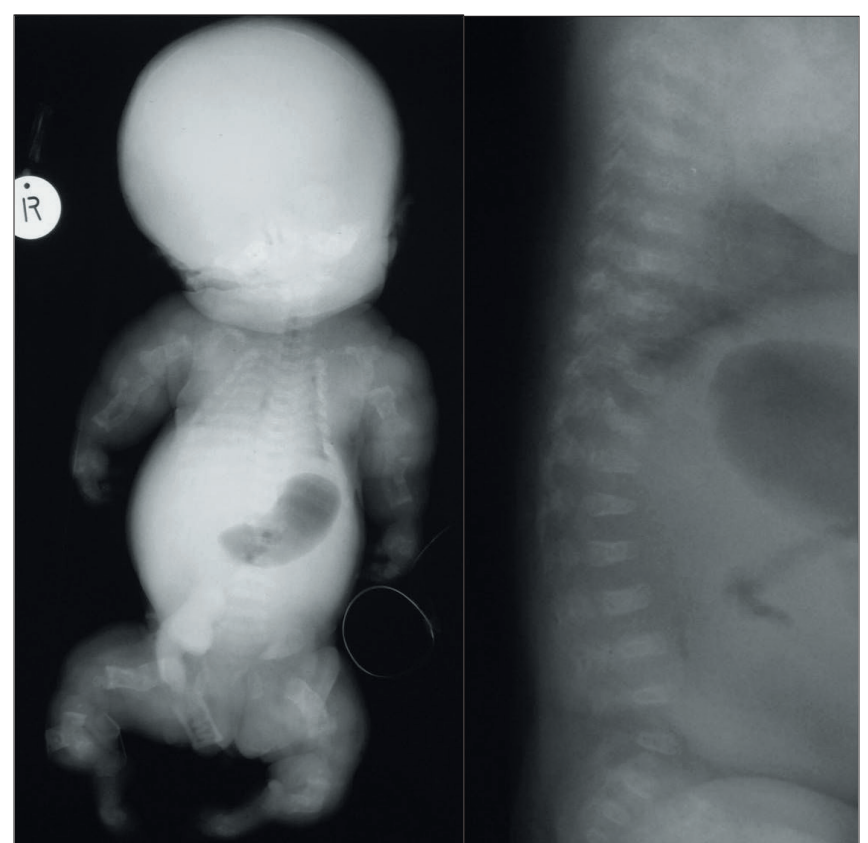

Fig. 2(a). Babygram X-ray of Patient 1 demonstrating generalised undermineralisation. The skull bones are poorly ossified and facial bones poorly mineralised. The ribs are broad with continuous beading. All long bones are short and broad with crumpled shafts.

Fig. 2(b). The spine of Patient 1 shows generalised mild platyspondyly, with irregularly shaped vertebral bodies.

in South Africa, the diagnosis of OI Type IIA relies on the accurate recognition of typical clinical and radiographic features. Parents can be reassured that the recurrence risk for future pregnancies is low, as most affected individuals have de novo mutations.

\section{Clinical findings}

The clinical findings in Patient 1 are seen in Fig. 1. Patient 2 was a male fetus, noted to have a small chest, short bowed long bones, and multiple fractures on antenatal ultrasound. The parents opted to interrupt the pregnancy, based on the diagnosis of a lethal skeletal dysplasia and the associated poor prognosis. The fetus was delivered at 25 weeks' gestation. Dysmorphic features included: soft skull, prominent eyes with periorbital fullness, blue sclerae, micro-retrognathia and short extremities.

\section{Radiological findings}

Major radiological features of OI Type IIA may include severe retardation of calvarial bone formation (Fig. 2a), generalised undermineralisation with multiple fractures and callus formation, wavy accordion-like appearance of the femora as well as short, thick crumpled shafts of long bones (Figs 2a and 3) and short, thickened ribs with continuous beading (Fig. 3). ${ }^{2}$

\section{Discussion}

OI Type IIA is an autosomal dominant condition caused by mutations in the collagen 1 alpha- 1 chain (COL1A1) and collagen 1 alpha- 2 chain

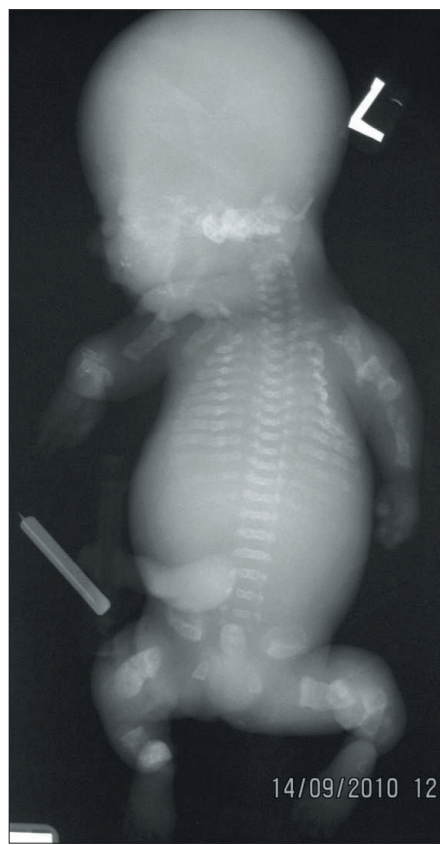

Fig. 3. Babygram X-ray of Patient 2 demonstrating generalised undermineralisation and evidence of multiple fractures. The skull is poorly ossified. The ribs show continuous beading. All long bones are short and broad with crumpled shafts.

(COL1A2) genes. They encode the two chains pro a1(I) and pro $\alpha 2(\mathrm{I})$, respectively, of Type I procollagen.

As molecular genetic testing for OI Type 2 is not currently available in South Africa, it is important to recognise the clinical and $\mathrm{X}$-ray findings. An assessment by a medical geneticist is recommended to confirm the diagnosis. An accurate clinico-radiological diagnosis is important, as it has direct implications for the family concerned. Firstly, the poor prognosis can be explained to the family, as OI Type IIA is invariably lethal. Secondly, it is well known that virtually all individuals with OI Type IIA have de novo mutations. Although the recurrence risk is never zero, owing to possible germline mosaicism in an unaffected parent, families can be reassured that the risk for future pregnancies is very low. Consequently, we would advise that all parents be referred for genetic counselling, for explaining the condition and reinforcing the expected low risks for future pregnancies.

Ethics approval was obtained from University of the Witwatersrand Human Research Ethics Committee (medical): Certificate M120152. Written consent from the parents was obtained for use of the images.

1. Online Mendelian Inheritance in Man, OMIM. Johns Hopkins University, Baltimore, MD. MIM Number: 166210: 06/10/2011. http://omim.org (accessed 1 September 2012).

2. Spranger JW, Brill PW, Poznanski A. Bone Dysplasias: An Atlas of Genetic Disorders of Skeletal Development. 2nd ed. Oxford: Oxford University Press, 2002. 